ANNALES

POLONICI MATHEMATICI

$85.1(2005)$

\title{
Theorems of Thron's type for random-valued vector functions and the Krein-Rutman theorem
}

\author{
by RAFAŁ KAPICA (Katowice)
}

\begin{abstract}
We propose stochastic versions of some theorems of W. J. Thron [14] on the speed of convergence of iterates for random-valued functions on cones in Banach spaces.
\end{abstract}

1. Introduction. In the deterministic case we have very useful theorems of W. J. Thron [14] which say how fast the sequence of iterates converges to zero for functions of the form

$$
f(x)=s x+x^{1+\alpha} F(x),
$$

where $\alpha$ is a positive number, $F$ is a bounded function and $s \in[0,1]$. An attempt to get stochastic versions of Thron's theorems was made in [2] for scalar functions; cf. also [11]. In the deterministic case some versions of Thron's theorem for more general spaces were obtained in [13], [15] and [16]. In the present paper we examine the speed of convergence of iterates of random-valued vector functions. We follow the general idea of J. Walorski to apply the theorem of Krein-Rutman. We use part of his reasoning presented in [15] and [16] (cf. also [6]). The iterates of random-valued functions were defined independently in [4] and [5] and then studied also in [2], [11] in the scalar case and in [8] in the vector case. They are useful for instance in solving functional-integral equations (see, e.g., [3], [7] and [9]).

Fix a Banach lattice $E$ and a compact linear operator $L: E \rightarrow E$, denote by $E_{+}$the positive cone of $E$, i.e. $E_{+}=\{x \in E: x \geq 0\}$, and assume that Int $E_{+} \neq \emptyset$,

$$
L E_{+} \subset E_{+}
$$

and for every $x>0$ there exists a positive integer $n$ with $L^{n} x \in \operatorname{Int} E_{+}$.

Let $\varrho$ denote the spectral radius of $L$. According to the Krein-Rutman theorem [10; Theorem 6.3] there are uniquely determined $u \in \operatorname{Int} E_{+}$and

2000 Mathematics Subject Classification: Primary 39B12; Secondary 37H99, 60F15.

Key words and phrases: random-valued vector functions, sequences of iterates, speed of convergence. 
$x^{*} \in E^{*}$ for which

$$
\begin{gathered}
L u=\varrho u, \quad\|u\|=1, \\
x^{*} L x=\varrho x^{*} x \quad \text { for } x \in E, \\
x^{*} u=1, \quad x^{*} x>0 \quad \text { for } x>0 ;
\end{gathered}
$$

moreover (see [10; pp. 269-270]) the spectral radius of the operator $S$ : $E \rightarrow E$ defined by

$$
S x=L x-\varrho u x^{*} x
$$

is strictly less than $\varrho$, and

$$
\left\|\varrho^{-n} L^{n} x-u x^{*} x\right\| \leq \varrho^{-n}\left\|S^{n}\right\|\|x\| \quad \text { for } x \in E \text { and } n \in \mathbb{N} .
$$

Consider a subset $X$ of $E_{+}$such that $0 \in \operatorname{cl} X \backslash X$ and, for $n \in \mathbb{N}$, an arbitrary nonempty set $\Omega_{n}$. Let $\varphi_{n}: X \times \Omega_{n} \rightarrow X$ be functions such that

$$
\lim _{x \rightarrow 0} \sup _{n \in \mathbb{N}} \sup _{\omega \in \Omega_{n}} \frac{\left\|\varphi_{n}(x, \omega)-L x\right\|}{\|x\|}=0 .
$$

Moreover assume that there exists a positive constant $c$ such that

$$
x^{*} x \geq c\left\|x^{*}\right\|\|x\| \quad \text { for } x \in X_{0}:=\bigcup_{n=1}^{\infty} \varphi_{n}\left(X \times \Omega_{n}\right) .
$$

REMARK 1.1. If $E$ is finite-dimensional, then $c$ defined by

$$
c=\inf \left\{\frac{x^{*} x}{\|x\|}: x \in E_{+},\|x\|=1\right\}
$$

is positive and

$$
x^{*} x \geq c\left\|x^{*}\right\|\|x\| \quad \text { for } x \in E_{+} .
$$

In particular (1.3) holds.

REMARK 1.2 (cf. [10; p. 210, Lemma 1.2]). If $c$ is a positive constant such that the closed ball centered at $\varphi_{n}(x, \omega) /\left\|\varphi_{n}(x, \omega)\right\|$ with radius $c$ is contained in $E_{+}$for all $x \in X, \omega \in \Omega_{n}$ and $n \in \mathbb{N}$, then (1.3) holds.

EXAmple 1.1. Assume that $E$ is the Banach lattice of all continuous real functions on the unit interval $I$ with the supremum norm and the coordinatewise ordering, let $X=E_{+} \backslash\{0\}$ and $\Omega_{n}=\Omega$ for $n \in \mathbb{N}$. Fix a positive continuous function $\lambda$ on $I^{2}$ and define $L: E \rightarrow E$ by

$$
L x(t)=\int_{0}^{1} \lambda(s, t) x(s) d s .
$$

Clearly, $L$ is linear and compact. For every $\omega \in \Omega$ and $x \in X$ consider also a nonnegative and continuous function $\lambda_{x, \omega}$ on $I^{2}$. We assume that for some $\alpha>0$ the function

$$
x \mapsto \max \left\{1,\|x\|^{\alpha}\right\} \sup \left\{\lambda_{x, \omega}(s, t):(s, t) \in I^{2}, \omega \in \Omega\right\}, \quad x \in X,
$$


is bounded. Then the function $\varphi: X \times \Omega \rightarrow X$ defined by

$$
\varphi(x, \omega)(t)=L x(t)+\int_{0}^{1} \lambda_{x, \omega}(s, t) x(s)^{1+\alpha} d s
$$

satisfies

$$
\limsup _{x \rightarrow 0} \sup _{\omega \in \Omega} \frac{\|\varphi(x, \omega)-L x\|}{\|x\|^{1+\alpha}}<\infty
$$

and

$$
\frac{\varphi(x, \omega)}{\|\varphi(x, \omega)\|} \geq c \quad \text { for all } x \in X, \omega \in \Omega
$$

with a positive constant $c$. On account of Remark 1.2 we obtain

$$
x^{*} x \geq c\left\|x^{*}\right\|\|x\| \quad \text { for } x \in \varphi(X \times \Omega) .
$$

We shall examine the speed of convergence of the sequence $f^{n}: X \times \Omega^{\infty}$ $\rightarrow X$ defined by

$$
\begin{aligned}
& f^{0}\left(x, \omega_{1}, \omega_{2}, \ldots\right)=x \\
& f^{n}\left(x, \omega_{1}, \omega_{2}, \ldots\right)=\varphi_{n}\left(f^{n-1}\left(x, \omega_{1}, \omega_{2}, \ldots\right), \omega_{n}\right)
\end{aligned}
$$

for $x \in X$ and $\left(\omega_{1}, \omega_{2}, \ldots\right) \in \Omega^{\infty}:=\prod_{n=1}^{\infty} \Omega_{n}$.

In the special case where $\varphi_{1}=\varphi_{2}=\cdots=f$, we have the iterates $f^{n}$ of $f$ in the sense of [4] and [5]. Moreover, if $(\Omega, \mathcal{A})$ is a measure space and $f: X \times \Omega \rightarrow X$ is a random-valued (vector) function, i.e. it is measurable with respect to the product $\sigma$-algebra $\mathcal{B}(X) \otimes \mathcal{A}$, where $\mathcal{B}(X)$ denotes the $\sigma$-algebra of all Borel subsets of $X$, then (cf. Remark 3.1) $f^{n}: X \times \Omega^{\infty} \rightarrow X$ is a random-valued function on the product $\left(\Omega^{\infty}, \mathcal{A}^{\infty}\right)$. More exactly, the $n$th iterate $f^{n}$ is $\mathcal{B}(X) \otimes \mathcal{F}_{n}$-measurable, where $\mathcal{F}_{n}$ denotes the $\sigma$-algebra of all sets of the form

$$
\left\{\left(\omega_{1}, \omega_{2}, \ldots\right) \in \Omega^{\infty}:\left(\omega_{1}, \ldots, \omega_{n}\right) \in A\right\}
$$

with $A$ in the product $\sigma$-algebra $\mathcal{A}^{n}$. It may be shown that these iterates form a random dynamical system (see [1]). For their intuitive probabilistic description let us imagine a mechanism which for each discrete time $n$ throws a "die" in order to select a mapping $S_{n}$ according to which a point $x_{n}$ moves to $x_{n+1}=S_{n}\left(x_{n}\right)$. The selection mechanism is permitted to remember all past decisions. The only thing of importance is that at each step the same mechanism is applied. Clearly, it is the procedure connected with iterated function systems (see [12]): If $\Omega=\{1, \ldots, N\}$ and $\xi_{n}$ on $\Omega^{\infty}$ are given by $\xi_{n}\left(\omega_{1}, \omega_{2}, \ldots\right)=\omega_{n+1}$, then with $f(x, n)=S_{n}(x)$ we have

$$
x_{n+1}(\omega):=S_{\xi_{n}(\omega)}\left(x_{n}(\omega)\right)=f\left(x_{n}(\omega), \omega_{n+1}\right)=f^{n+1}\left(x_{0}, \omega\right) .
$$

In this special (but in fact the most important) case, in [8] some conditions are established which guarantee the convergence (a.s. and in $L^{1}$ ) 
of $\left(f^{n}(x, \cdot)\right)$. It is the aim of the present paper to examine the speed of this convergence.

2. Auxiliary results: the Joffe-Spitzer sequence. Following [15] (cf. also [6]), we consider the sequence

$$
\frac{f^{n}\left(x_{0}, \cdot\right)}{x^{*} f^{n}\left(x_{0}, \cdot\right)}
$$

which will be used in the main part of this paper. Here and in what follows, $x_{0}$ is an arbitrarily fixed point of $X$.

Proposition 2.1. Assume that either $\varrho<1$, or $\varrho=1$ and $\varphi_{n}(x, \omega)$ $\leq$ Lx for $x \in X, \omega \in \Omega_{n}, n \in \mathbb{N}$. If the sequence $\left(f^{n}\left(x_{0}, \cdot\right)\right)$ converges to zero uniformly on a nonempty subset $A$ of $\Omega^{\infty}$, then the sequence (2.1) converges to the eigenvector $u$ uniformly on $A$.

Proof. Let $r_{0}$ be a positive number such that the closed ball centered at $u$ and with radius $r_{0}$ is contained in the positive cone $E_{+}$. Then

$$
x \leq \frac{1}{r_{0}}\|x\| u \quad \text { for } x \in E .
$$

Put

$$
\alpha_{n}=\frac{\left\|S^{n}\right\|}{\varrho^{n} c r_{0}\left\|x^{*}\right\|} .
$$

Since the spectral radius of $S$ is less than $\varrho$ it follows that

$$
\lim _{n \rightarrow \infty} \alpha_{n}=0 \text {. }
$$

Moreover, from (2.2) and (1.1) we obtain

$$
\pm\left(\varrho^{n} u x^{*} x-L^{n} x\right) \leq \frac{1}{r_{0}}\left\|S^{n}\right\|\|x\| u
$$

which jointly with (1.3) gives

$$
\pm\left(\varrho^{n} u x^{*} x-L^{n} x\right) \leq \alpha_{n} \varrho^{n} u x^{*} x \quad \text { for } x \in X_{0} \text { and } n \in \mathbb{N} .
$$

Consider functions $F_{n}: X \times \Omega_{n} \rightarrow E$ and $f^{m, m+k}: X \times \Omega^{\infty} \rightarrow X$ given by

$$
F_{n}(x, \omega)=\varphi_{n}(x, \omega)-L x
$$

and

$$
f^{m, m}(x, \omega)=\varphi_{m}\left(x, \omega_{m}\right), f^{m, m+k}(x, \omega)=\varphi_{m+k}\left(f^{m, m+k-1}(x, \omega), \omega_{m+k}\right) .
$$

Then

$$
\begin{aligned}
f^{m, m+n}(x, \omega)= & L^{n} f^{m, m}(x, \omega) \\
& +\sum_{k=0}^{n-1} L^{n-k-1} F_{m+k+1}\left(f^{m, m+k}(x, \omega), \omega_{m+k+1}\right)
\end{aligned}
$$


and

$$
f^{m, m+n}\left(f^{m-1}(x, \omega), \omega\right)=f^{m+n}(x, \omega) .
$$

Applying (2.3) and (2.4) we get

$$
\begin{array}{r} 
\pm\left(\varrho^{n} u x^{*} f^{m, m}(x, \omega)\right. \\
\left.+\sum_{k=0}^{n-1} L^{n-k+1} F_{m+k+1}\left(f^{m, m+k}(x, \omega), \omega_{m+k+1}\right)-f^{m, m+n}(x, \omega)\right) \\
\leq \alpha_{n} \varrho^{n} u x^{*} f^{m, m}(x, \omega) .
\end{array}
$$

We shall show that there exists a positive integer $m_{0}$ such that

$$
x^{*} f^{m+k}\left(x_{0}, \omega\right) \leq x^{*} f^{m}\left(x_{0}, \omega\right) \quad \text { for } \omega \in A \text { and } m \geq m_{0} .
$$

To this end assume first that $\varrho<1$. Then there exists a $\delta>0$ such that

$$
\frac{\left\|F_{n}(x, \omega)\right\|}{\|x\|} \leq(1-\varrho) c r_{0}\left\|x^{*}\right\|
$$

for $\omega \in \Omega_{n}, n \in \mathbb{N}$ and $x \in X$ with $\|x\| \leq \delta$. Hence and from (1.3) we get

$$
\left\|F_{n}(x, \omega)\right\| \leq(1-\varrho) r_{0} x^{*} x
$$

for $\omega \in \Omega_{n}, n \in \mathbb{N}$ and $x \in X_{0}$ with $\|x\| \leq \delta$. Consequently, by (2.2)

$$
F_{n}(x, \omega) \leq(1-\varrho) u x^{*} x,
$$

whence

$$
x^{*} \varphi_{n}(x, \omega)=x^{*} L x+x^{*} F_{n}(x, \omega) \leq \varrho x^{*} x+(1-\varrho) x^{*} u x^{*} x=x^{*} x
$$

for $\omega \in \Omega_{n}, n \in \mathbb{N}$ and $x \in X_{0}$ with $\|x\| \leq \delta$. This shows that (2.7) holds whenever $m_{0}$ is a positive integer such that $\left\|f^{m}\left(x_{0}, \omega\right)\right\| \leq \delta$ for $m \geq m_{0}$ and $\omega \in A$. In the case where $\varrho=1$ and $\varphi_{n}(x, \omega) \leq L x$ for $x \in X, \omega \in \Omega_{n}$ and $n \in \mathbb{N}$ we have

$$
x^{*} \varphi_{n}(x, \omega) \leq x^{*} x
$$

for all $x \in X$ and so it is enough to take $m_{0}=1$.

Define now functions $\beta_{n}: \Omega^{\infty} \rightarrow[0, \infty)$ by

$$
\beta_{n}(\omega)=\frac{\left\|F_{n+1}\left(f^{n}\left(x_{0}, \omega\right), \omega_{n+1}\right)\right\|}{c r_{0}\left\|x^{*}\right\|\left\|f^{n}\left(x_{0}, \omega\right)\right\|} .
$$

Since the sequence $\left(f^{n}\left(x_{0}, \cdot\right)\right)$ is uniformly convergent on the set $A$, from (1.2) it follows that $\left(\beta_{n}\right)$ converges to zero uniformly on $A$. Moreover, from (2.2) and (1.3) we have

$$
\begin{aligned}
\pm F_{m+k+1}\left(f^{m+k}\left(x_{0}, \omega\right), \omega_{m+k+1}\right) & \leq \beta_{m+k}(\omega)\left\|f^{m+k}\left(x_{0}, \omega\right)\right\| c\left\|x^{*}\right\| u \\
& \leq \beta_{m+k}(\omega) u x^{*} f^{m+k}\left(x_{0}, \omega\right) .
\end{aligned}
$$


Consequently, the monotonicity of $L$ yields

$$
\begin{aligned}
\pm\left(L ^ { n - k - 1 } F _ { m + k + 1 } \left(f^{m+k}\left(x_{0}, \omega\right),\right.\right. & \left.\left.\omega_{m+k+1}\right)\right) \\
& \leq \varrho^{n-k-1} u \beta_{m+k}(\omega) x^{*} f^{m+k}\left(x_{0}, \omega\right)
\end{aligned}
$$

on $\Omega^{\infty}$. This jointly with (2.5), (2.6) and (2.7) gives

$$
\begin{aligned}
& \pm\left(\varrho^{n} u x^{*} f^{m}\left(x_{0}, \omega\right)-f^{m+n}\left(x_{0}, \omega\right)\right) \\
& \quad \leq \varrho^{n} \alpha_{n} u x^{*} f^{m}\left(x_{0}, \omega\right)+\sum_{k=0}^{n-1} \varrho^{n-k-1} u \beta_{m+k}(\omega) x^{*} f^{m}\left(x_{0}, \omega\right),
\end{aligned}
$$

whence

$$
\begin{aligned}
\left(\varrho^{n}\left(1-\alpha_{n}\right)-\right. & \left.\sum_{k=0}^{n-1} \varrho^{n-k-1} \beta_{m+k}(\omega)\right) u x^{*} f^{m}\left(x_{0}, \omega\right) \leq f^{m+n}\left(x_{0}, \omega\right) \\
& \leq\left(\varrho^{n}\left(1+\alpha_{n}\right)+\sum_{k=0}^{n-1} \varrho^{n-k-1} \beta_{m+k+1}(\omega)\right) u x^{*} f^{m}\left(x_{0}, \omega\right)
\end{aligned}
$$

and

$$
\begin{aligned}
& \left(\varrho^{n}\left(1-\alpha_{n}\right)-\sum_{k=0}^{n-1} \beta_{m+k}(\omega)\right) x^{*} f^{m}\left(x_{0}, \omega\right) \leq x^{*} f^{m+n}\left(x_{0}, \omega\right) \\
& \quad \leq\left(\varrho^{n}\left(1+\alpha_{n}\right)+\sum_{k=0}^{n-1} \varrho^{n-k-1} \beta_{m+k}(\omega)\right) x^{*} f^{m}\left(x_{0}, \omega\right)
\end{aligned}
$$

for $m \geq m_{0}$ and $\omega \in A$. Let $n_{0}$ be a positive integer such that $\alpha_{n}<1$ for $n \geq n_{0}$, and for every $n \geq n_{0}$ let $m_{n} \geq m_{0}$ be an integer such that

$$
\sup _{\omega \in A} \sum_{k=0}^{n-1} \varrho^{-k-1} \beta_{m+k}(\omega)<1-\alpha_{n} \quad \text { for } m \geq m_{n} .
$$

Then

$$
\begin{array}{r}
-2 \frac{\alpha_{n}+\sum_{k=0}^{n-1} \varrho^{-k-1} \beta_{m+k}(\omega)}{1-\alpha_{n}-\sum_{k=0}^{n-1} \varrho^{-k-1} \beta_{m+k}(\omega)} \leq-2 \frac{\alpha_{n}+\sum_{k=0}^{n-1} \varrho^{-k-1} \beta_{m+k}(\omega)}{1+\alpha_{n}+\sum_{k=0}^{n-1} \varrho^{-k-1} \beta_{m+k}(\omega)} \\
\leq \frac{f^{m+n}\left(x_{0}, \omega\right)}{x^{*} f^{m+n}\left(x_{0}, \omega\right)} u \leq 2 \frac{\alpha_{n}+\sum_{k=0}^{n-1} \varrho^{-k-1} \beta_{m+k}(\omega)}{1-\alpha_{n}-\sum_{k=0}^{n-1} \varrho^{-k-1} \beta_{m+k}(\omega)}
\end{array}
$$

and, consequently,

$$
\left\|\frac{f^{m+n}\left(x_{0}, \omega\right)}{x^{*} f^{m+n}\left(x_{0}, \omega\right)}-u\right\| \leq 6 \frac{\alpha_{n}+\sum_{k=0}^{n-1} \varrho^{-k-1} \beta_{m+k}(\omega)}{1-\alpha_{n}-\sum_{k=0}^{n-1} \varrho^{-k-1} \beta_{m+k}(\omega)}
$$

for $m \geq m_{n}$ and $n \geq n_{0}$, which ends the proof.

Corollary 2.1. Assume that either $\varrho<1$, or $\varrho=1$ and $\varphi_{n}(x, \omega) \leq L x$ for $x \in X, \omega \in \Omega_{n}, n \in \mathbb{N}$. If the sequence $\left(f^{n}\left(x_{0}, \cdot\right)\right)$ converges to zero 
uniformly on a nonempty subset $A$ of $\Omega^{\infty}$, then the sequence

$$
\left(\frac{f^{n}\left(x_{0}, \cdot\right)}{\left\|f^{n}\left(x_{0}, \cdot\right)\right\|}\right)
$$

converges to $u$ uniformly on $A$ and the sequence

$$
\left(\frac{\left\|f^{n+1}\left(x_{0}, \cdot\right)\right\|}{\left\|f^{n}\left(x_{0}, \cdot\right)\right\|}\right)
$$

converges to @ uniformly on $A$.

Proof. The first part follows immediately from Proposition 2.1. To get the second one it is enough to observe that

$$
\frac{\left\|f^{n+1}\left(x_{0}, \omega\right)\right\|}{\left\|f^{n}\left(x_{0}, \omega\right)\right\|}=\frac{\left\|f^{n+1}\left(x_{0}, \omega\right)\right\|}{x^{*} f^{n+1}\left(x_{0}, \omega\right)} \frac{x^{*} f^{n+1}\left(x_{0}, \omega\right)}{x^{*} f^{n}\left(x_{0}, \omega\right)} \frac{x^{*} f^{n}\left(x_{0}, \omega\right)}{\left\|f^{n}\left(x_{0}, \omega\right)\right\|}
$$

and

$$
\begin{aligned}
\lim _{x \in X_{0}, x \rightarrow 0} \sup _{n \in \mathbb{N}} \sup _{\omega \in \Omega_{n}} \frac{x^{*} \varphi_{n}(x, \omega)}{x^{*} x} \\
\quad=\lim _{x \in X_{0}, x \rightarrow 0} \sup _{n \in \mathbb{N}} \sup _{\omega \in \Omega_{n}}\left(\varrho+x^{*} \frac{\varphi_{n}(x, \omega)-L x}{\|x\|} \frac{\|x\|}{x^{*} x}\right)=0
\end{aligned}
$$

and to apply Proposition 2.1 again.

3. Main theorems. We start with the following analogue of [16; Theorem 2] concerning the sequence

$$
\frac{f^{n}\left(x_{0}, \cdot\right)}{\varrho^{n}} .
$$

TheOREM 3.1. Assume that $\varrho<1$ and there exists a positive constant $\alpha$ such that

$$
\limsup _{x \rightarrow 0} \sup _{n \in \mathbb{N}} \sup _{\omega \in \Omega_{n}} \frac{\left\|\varphi_{n}(x, \omega)-L x\right\|}{\|x\|^{1+\alpha}}<\infty .
$$

If the sequence $\left(f^{n}\left(x_{0}, \cdot\right)\right)$ converges to zero uniformly on a nonempty subset $A$ of $\Omega^{\infty}$, then there exists a bounded function $\xi: A \rightarrow(0, \infty)$ such that the sequence (3.1) converges to $\xi u$ uniformly on $A$.

Proof. According to Proposition 2.1 it is enough to show that the sequence

$$
\frac{x^{*} f^{n}\left(x_{0}, \cdot\right)}{\varrho^{n}}
$$

converges uniformly on $A$ to a bounded function $\xi$. Let

$$
\beta_{n}(\omega)=\frac{x^{*}\left(f^{n+1}\left(x_{0}, \omega\right)-L f^{n}\left(x_{0}, \omega\right)\right)}{x^{*} f^{n}\left(x_{0}, \omega\right)^{1+\alpha}}, \quad \gamma_{n}(\omega)=x^{*} f^{n}\left(x_{0}, \omega\right) .
$$


It follows from (3.2) and Proposition 2.1 that $\sup _{n \geq N} \sup _{\omega \in A}\left|\beta_{n}(\omega)\right|$ is finite for large $N$. Moreover,

$$
\frac{\gamma_{n+1}(\omega)}{\gamma_{n}(\omega)}=\varrho+\gamma_{n}^{\alpha}(\omega) \beta_{n}(\omega)
$$

Hence the series $\sum_{n=1}^{\infty} \gamma_{n}^{\alpha}$ converges uniformly on $A$ and

$$
\frac{\gamma_{n}}{\varrho^{n}}=\gamma_{0} \prod_{k=0}^{n-1}\left(1+\frac{\gamma_{k}^{\alpha} \beta_{k}}{\varrho}\right)
$$

Consequently, $\left(\varrho^{-n} \gamma_{n}\right)$ converges uniformly on $A$ to a bounded function as desired.

The following shows a possible realization of the assumptions of Theorem 3.1 .

ExAmPlE 3.1. Let $0 \leq a<b<\infty$, consider the function $\varphi$ defined in Example 1.1 with $\Omega=[a, b]$ and

$$
\lambda_{x, \omega}(s, t)=\frac{\omega x(s)}{\max \left\{1,\|x\|^{\alpha}\right\}(1+x(s))}
$$

and put $\varphi_{n}=\varphi$ for $n \in \mathbb{N}$. Setting $\eta=\max \lambda\left(I^{2}\right)$ we have

$$
0 \leq \varphi(x, \omega) \leq(\eta+b) \int_{0}^{1} x(s) d s \quad \text { for } x \in X \text { and } \omega \in[a, b],
$$

whence

$$
\left\|f^{n}(x, \omega)\right\| \leq(\eta+b)^{n} \int_{0}^{1} x(s) d s \quad \text { for } x \in X, \omega \in[a, b] \text { and } n \in \mathbb{N} .
$$

Consequently, if $\eta+b<1$, then $\left(f^{n}(x, \cdot)\right)$ converges to zero uniformly on $\Omega^{\infty}$. It follows from Theorem 3.1 that there exists a (bounded) function $\xi: \Omega^{\infty} \rightarrow(0, \infty)$ such that the sequence (3.1) converges to $\xi u$ uniformly on $\Omega^{\infty}$. Due to the continuity of the function (3.1), the function $\xi$ is in fact continuous. In particular, $\xi\left(\Omega^{\infty}\right)$ is a compact interval.

Assume now that we also have a finite measure on a $\sigma$-algebra of subsets of $\Omega^{\infty}$. Applying Egorov's Theorem we obtain from Theorem 3.1 the following corollary.

Corollary 3.1. Suppose that $\varrho<1$ and (3.2) holds with a positive constant $\alpha$. If $\left(f^{n}\left(x_{0}, \cdot\right)\right)$ is a sequence of measurable functions which converges a.s. to zero, then there exists a measurable function $\xi: \Omega^{\infty} \rightarrow(0, \infty)$ such that the sequence (3.1) converges a.s. to $\xi u$.

REMARK 3.1. Suppose that $\left(\Omega_{n}, \mathcal{A}_{n}\right)$ is a measure space for every $n \in \mathbb{N}$ and consider the product $\sigma$-algebra $\bigotimes_{n=1}^{\infty} \mathcal{A}_{n}$ in $\Omega^{\infty}$ and the $\sigma$-algebra $\mathcal{B}(X)$ 
of all Borel subsets of $X$. If $\varphi_{n}: X \times \Omega_{n} \rightarrow X$ are measurable with respect to the product $\sigma$-algebra $\mathcal{B}(X) \otimes \mathcal{A}_{n}$, then (cf. [2; Lemma 3], [8]) the iterates $f^{n}: X \times \Omega^{\infty} \rightarrow X$ are measurable with respect to $\mathcal{B}(X) \otimes \bigotimes_{n=1}^{\infty} \mathcal{A}_{n}$.

Passing to the case where $\varrho=1$ we assume that $\Omega_{n}$ is a probability space for every $n \in \mathbb{N}$ and in the product $\Omega^{\infty}$ we consider both the product $\sigma$-algebra and the product probability measure. In this case we have the following result.

Theorem 3.2. Assume that $\varrho=1$ and $\varphi_{n}(x, \omega) \leq L x$ for every $x \in X$, $\omega \in \Omega_{n}$ and $n \in \mathbb{N}$. Suppose also that $\alpha$ is a positive constant and $\left(\Phi_{n}\right)$ is a sequence of function $\Phi_{n}: \Omega_{n} \rightarrow E_{+}$such that $x^{*} \Phi_{n}$ is measurable, $\sup _{n \in \mathbb{N}} \operatorname{ess} \sup \left|x^{*} \Phi_{n}\right|<\infty$ and

$$
\lim _{x \rightarrow 0} \sup _{n \in \mathbb{N}} \sup _{\omega \in \Omega_{n}}\left\|\frac{\varphi_{n}(x, \omega)-L x}{\|x\|^{1+\alpha}}+\Phi_{n}(\omega)\right\|=0 .
$$

If $\lim _{n \rightarrow \infty} f^{n}\left(x_{0}, \cdot\right)=0$ a.s., then

$$
\lim _{n \rightarrow \infty}\left(\frac{1}{n\left(x^{*} f^{n}\left(x_{0}, \cdot\right)\right)^{\alpha}}-\frac{\alpha}{n} \sum_{k=1}^{n} E x^{*} \Phi_{k}\right)=0 \quad \text { a.s. }
$$

Moreover, if additionally

$$
\liminf _{n \rightarrow \infty} \frac{1}{n} \sum_{k=1}^{n} E x^{*} \Phi_{k}>0,
$$

then

$$
\lim _{n \rightarrow \infty}\left(n^{1 / \alpha} f^{n}\left(x_{0}, \cdot\right)-\left(\frac{\alpha}{n} \sum_{k=1}^{n} E x^{*} \Phi_{k}\right)^{-1 / \alpha} u\right)=0 \quad \text { a.s. }
$$

Proof. Let $M$ be a positive constant such that

$$
\left|(1+t)^{\alpha}-1-\alpha t\right| \leq M t^{2} \quad \text { for } t \in[0,1] .
$$

Fix an $\omega \in \Omega^{\infty}$ such that

$$
\lim _{n \rightarrow \infty} f^{n}\left(x_{0}, \omega\right)=0, \quad \sup _{n \in \mathbb{N}}\left|x^{*} \Phi_{n}\left(\omega_{n}\right)\right|<\infty
$$

and

$$
\lim _{n \rightarrow \infty} \frac{1}{n} \sum_{k=1}^{n}\left(x^{*} \Phi_{k}\left(\omega_{k}\right)-E x^{*} \Phi_{k}\right)=0,
$$

and for this $\omega$ define $\beta_{n}$ and $\gamma_{n}$ as in the proof of Theorem 3.1. Then

$$
\frac{\gamma_{n+1}}{\gamma_{n}}=1+\gamma_{n}^{\alpha} \beta_{n},
$$


whence

$$
\frac{\gamma_{n}^{\alpha}}{\gamma_{n+1}^{\alpha}}=\left(1-\frac{\gamma_{n}^{\alpha} \beta_{n}}{1+\gamma_{n}^{\alpha} \beta_{n}}\right)^{\alpha} \text { and } \frac{\gamma_{n}^{\alpha} \beta_{n}}{1+\gamma_{n}^{\alpha} \beta_{n}} \leq 0
$$

for all $n \in \mathbb{N}$. On the other hand,

$$
\begin{aligned}
\left|\beta_{n}+x^{*} \Phi_{n+1}\left(\omega_{n+1}\right)\right| \leq & \left|x^{*} \Phi_{n+1}\left(\omega_{n+1}\right)\right|\left(1-\left(\frac{\left\|f^{n}\left(x_{0}, \omega\right)\right\|}{x^{*} f^{n}\left(x_{0}, \omega\right)}\right)^{1+\alpha}\right) \\
& +\left(\frac{\left\|f^{n}\left(x_{0}, \omega\right)\right\|}{x^{*} f^{n}\left(x_{0}, \omega\right)}\right)^{1+\alpha}\left\|x^{*}\right\| \\
\times & \left\|\frac{\varphi_{n+1}\left(f^{n}\left(x_{0}, \omega\right), \omega_{n+1}\right)-L f^{n}\left(x_{0}, \omega\right)}{\left\|f^{n}\left(x_{0}, \omega\right)\right\|^{1+\alpha}}+\Phi_{n+1}\left(\omega_{n+1}\right)\right\| .
\end{aligned}
$$

From (3.3) and Proposition 2.1, we now get

$$
\lim _{n \rightarrow \infty}\left(\beta_{n}+x^{*} \Phi_{n+1}\left(\omega_{n+1}\right)\right)=0 .
$$

Consequently, if $\varepsilon>0$ is fixed, then for $n$ large enough, say $n \geq n_{0}$, we have

$$
M \frac{\gamma_{n}^{\alpha} \beta_{n}^{2}}{\left(1+\gamma_{n}^{\alpha} \beta_{n}\right)^{2}} \leq \alpha \varepsilon \quad \text { and } \quad\left|\frac{\beta_{n}}{1+\gamma_{n}^{\alpha} \beta_{n}}+x^{*} \Phi_{n+1}\left(\omega_{n+1}\right)\right| \leq \varepsilon .
$$

As $\left(\frac{\gamma_{n}^{\alpha} \beta_{n}}{1+\gamma_{n}^{\alpha} \beta_{n}}\right)$ converges to zero, from (3.7) and (3.8) we infer that there exists a positive integer $n_{1} \geq n_{0}$ such that

$$
\left|\frac{\gamma_{n}^{\alpha}}{\gamma_{n+1}^{\alpha}}-1+\alpha \frac{\gamma_{n}^{\alpha} \beta_{n}}{1+\gamma_{n}^{\alpha} \beta_{n}}\right| \leq M\left(\frac{\gamma_{n}^{\alpha} \beta_{n}}{1+\gamma_{n}^{\alpha} \beta_{n}}\right)^{2}
$$

for $n \geq n_{1}$. Therefore by (3.9) we obtain

$$
\frac{1}{\gamma_{n}^{\alpha}}+\alpha x^{*} \Phi_{n+1}\left(\omega_{n+1}\right)-2 \alpha \varepsilon \leq \frac{1}{\gamma_{n+1}^{\alpha}} \leq \frac{1}{\gamma_{n}^{\alpha}}+\alpha x^{*} \Phi_{n+1}\left(\omega_{n+1}\right)+2 \alpha \varepsilon
$$

for $n \geq n_{1}$. This gives

$$
\begin{aligned}
\frac{1}{\gamma_{n_{0}}^{\alpha}}+\alpha \sum_{k=n_{0}+1}^{n} x^{*} \Phi_{k}\left(\omega_{k}\right)-2 & \alpha \varepsilon\left(n-n_{0}\right) \leq \frac{1}{\gamma_{n}^{\alpha}} \\
\leq & \frac{1}{\gamma_{n_{0}}^{\alpha}}+\alpha \sum_{k=n_{0}+1}^{n} x^{*} \Phi_{k}\left(\omega_{k}\right)+2 \alpha \varepsilon\left(n-n_{0}\right)
\end{aligned}
$$

for $n>n_{1}$, whence (3.4) easily follows.

To get (3.6) put

$$
x_{n}=f^{n}\left(x_{0}, \omega\right), \quad \delta_{n}=\left(\frac{\alpha}{n} \sum_{k=1}^{n} E x^{*} \Phi_{k}\right)^{-1 / \alpha} .
$$


Then, as we have just proved,

$$
\lim _{n \rightarrow \infty}\left(\left(n^{1 / \alpha} x^{*} x_{n}\right)^{-\alpha}-\delta_{n}^{-\alpha}\right)=0 .
$$

This jointly with (3.5) shows that

$$
\lim _{n \rightarrow \infty}\left(n^{1 / \alpha} x^{*} x_{n}-\delta_{n}\right)=0 .
$$

Moreover, applying Proposition 2.1 and the boundedness of $\left(\delta_{n}\right)$ we get

$$
\lim _{n \rightarrow \infty}\left(n^{1 / \alpha} x_{n}-\delta_{n} u\right)=0,
$$

which ends the proof.

Assume now that $\Omega$ is a probability space and $f$ maps $X \times \Omega$ into $X$. Defining the sequence $\left(f^{n}\right)$ of mappings $X \times \Omega^{\infty} \rightarrow X$ by

$$
f^{0}(x, \omega)=x, \quad f^{n}(x, \omega)=f\left(f^{n-1}(x, \omega), \omega_{n}\right)
$$

we obtain the following corollary concerning the case $\varrho=1$; cf. [2; Theorem 3] and [16; Theorem 3].

Corollary 3.2. Assume that $\varrho=1$ and $f(x, \omega) \leq L x$ for $x \in X$ and $\omega \in \Omega$. Suppose also that $\alpha$ is a positive constant and $\Phi: \Omega \rightarrow E_{+}$is a function such that $x^{*} \Phi$ is measurable, essentially bounded, not concentrated at zero and

$$
\lim _{x \rightarrow 0} \sup _{\omega \in \Omega}\left\|\frac{f(x, \omega)-L x}{\|x\|^{1+\alpha}}+\Phi(\omega)\right\|=0 .
$$

If $\lim _{n \rightarrow \infty} f^{n}\left(x_{0}, \cdot\right)=0$ a.s., then

$$
\lim _{n \rightarrow \infty} n^{1 / \alpha} f^{n}\left(x_{0}, \cdot\right)=\left(\alpha E x^{*} \Phi\right)^{-1 / \alpha} u \quad \text { a.s. }
$$

Acknowledgments. This research was supported by the Silesian University, Mathematics Department (Iterative Functional Equations and Real Analysis program).

\section{References}

[1] L. Arnold, Random Dynamical Systems, Lecture Notes in Math. 1609, Springer, Berlin, 1995.

[2] K. Baron, On the convergence of sequences on iterates of random-valued functions, Aequationes Math. 32 (1987), 240-251.

[3] K. Baron and W. Jarczyk, Random-valued functions and iterative functional equations, ibid. 67 (2004), 140-153.

[4] K. Baron and M. Kuczma, Iteration of random-valued functions on the unit interval, Colloq. Math. 37 (1977), 263-269.

[5] Ph. Diamond, A stochastic functional equation, Aequationes Math. 15 (1977), 225-233. 
[6] A. Joffe and F. Spitzer, On multitype branching processes with $\varrho \leq 1$, J. Math. Anal. Appl. 19 (1967), 409-430.

[7] R. Kapica, Sequences of iterates of random-valued vector functions and continuous solutions of a linear functional equation of infinite order, Bull. Polish Acad. Sci. Math. 50 (2002), 447-455.

[8] - Convergence of sequences of iterates of random-valued vector functions, Colloq. Math. 97 (2003), 1-6.

[9] -, Sequences of iterates of random-valued vector functions and solutions of related equations, Österreich. Akad. Wiss. Math.-Natur. Kl. Sitzungsber. II, to appear.

[10] M. G. Kreĭn and M. A. Rutman, Linear operators leaving invariant a cone in a Banach space, Uspekhi Mat. Nauk (N.S.) 3 (1948), no. 1, 3-95; English transl.: Functional Analysis and Measure Theory, Amer. Math. Soc. Transl. 10, 1962, 199325 .

[11] M. Kuczma, Normalizing factors for iterates of random valued functions, Uniw. Śląski w Katowicach, Prace Nauk.-Prace Mat. 6 (1975), 67-72.

[12] A. Lasota and M. C. Mackey, Chaos, Fractals, and Noise. Stochastic Aspects of Dynamics, Springer, New York, 1994.

[13] P. V. Subrahmanyam, On the convergence of iterates, Nonlinear Anal. 4 (1980), $1203-1211$.

[14] W. J. Thron, Sequences generated by iteration, Trans. Amer. Math. Soc. 96 (1960), $38-53$.

[15] J. Walorski, Convex solutions of the Schröder equation in Banach spaces, Proc. Amer. Math. Soc. 125 (1997), 153-158.

[16] —, Thron's theorems in Banach spaces, Grazer Math. Ber. 334 (1997), 233-240.

Institute of Mathematics

Silesian University

Bankowa 14

40-007 Katowice, Poland

E-mail: rkapica@ux2.math.us.edu.pl

Reçu par la Rédaction le 18.8.2003

Révisé le 20.10.2004 